\title{
Comparative analysis of various donor cell types for somatic cell nuclear transfer and its association with apoptosis and senescence
}

\author{
EUNHYE KIM and SANG-HWAN HYUN \\ Laboratory of Veterinary Embryology and Biotechnology, College of Veterinary Medicine, \\ Chungbuk National University, Cheongju, Chungbuk 361-763, Republic of Korea
}

Received August 1, 2013; Accepted October 25, 2013

DOI: $10.3892 / \mathrm{mmr} .2013 .1776$

\begin{abstract}
The aim of the present study was to characterize potential somatic cell nuclear transfer (SCNT) donor cells by comparing two lines of transfected cells with their non-modified parental controls in culture. Fetal fibroblasts used in the study originated from crossbred Landrace x Yorkshire x Duroc (LYD) or Yucatan mini-pigs. The LYD fibroblasts were modified by the transfection of a tetracycline on/off gene, whereas Yucatan fibroblasts were triple transfected with the complement regulatory factors, human decay-accelerating factor and human CD59, as well as H-transferase. At the 9th doubling passage, parameters associated with senescence and apoptosis, including morphology, mRNA expression (TP53, Bcl-2, Bax) and reactive oxygen species (ROS) levels, were evaluated. Population doubling (PD) time was calculated by assessing the time required for cell numbers to double by averaging the three cell passages. Quantitative polymerase chain reaction revealed that when comparing LYD with Yucatan fibroblasts, the latter exhibited a lower relative expression of TP53 and a higher relative expression of antiproliferative $\mathrm{Bcl}-2$, which correlated with the PD time results (26 and $40 \mathrm{~h}$, respectively). Tetracycline on/off transfected cell lines exhibited a lower relative expression of antiapoptotic $B c l-2$ compared with their originating LYD cells. Similarly, triple transgenic cells exhibited higher TP53 and Bax mRNA expression levels than their non-transgenic counterparts. For ROS measurement, cells were incubated with $2^{\prime}, 7^{\prime}$-dichlorofluorescin diacetate under the same conditions and were analyzed by flow cytometry. Yucatan fibroblasts exhibited higher ROS content than LYD cells. In addition, the two transgenic cell lines produced higher ROS levels than their corresponding non-transfected cell lines. In conclusion, these results indicate that characteristics associ-
\end{abstract}

Correspondence to: Professor Sang-Hwan Hyun, Laboratory of Veterinary Embryology and Biotechnology, College of Veterinary Medicine, Chungbuk National University, 52 Naesudong-ro, Cheongju, Chungbuk 361-763, Repulic of Korea

E-mail: shhyun@cbu.ac.kr

Key words: translational medicine, transgenic pigs, somatic cell nuclear transfer, donor cells, apoptosis, senescence ated with senescence and apoptosis in transfected cells during culture may affect the efficiency of SCNT when used as donor cells for the production of transgenic swine.

\section{Introduction}

Various cell types have been used as donors in somatic cell nuclear transfer (SCNT) technology to produce cloned animals from various species (1-4). While previous studies have indicated that the origin of the donor cell used in the cloning procedure is an important determinant of the developmental competence of SCNT embryos $(5,6)$, there is limited information available with regard to the donor cell effect on porcine cloning efficiency.

In addition, the efficiency of producing transgenic cloned animals depends on improving the combination of technologies involved, with the preparation and induction of competent transfected donor cells being a crucial upstream step. However, the genetic modification of donor cells requires long-term culture for adequate cell line establishment, followed by the time required for the transfection procedure. Therefore, the effects of apoptosis and senescence stemming from long-term donor cell culture on SCNT efficiency remain controversial $(7,8)$ and require further evaluation.

In order to address these issues, the present study used four cell lines, two transgenic cell lines and the corresponding non-transfected controls.

Following SCNT, donor cells transfected with a tetracycline on/off responsive gene provide a tool to control the expression of a specific gene at any time during development (9). Therefore, in the present study fibroblasts originating from crossbred Landrace $x$ Yorkshire $x$ Duroc (LYD) pig fibroblasts were transfected with a tetracycline responsive gene. In addition, since triple cell lines appear to be resistant to hyperacute rejection during xenotransplantation procedures (10), an additional fibroblast cell line was used originating from Yucatan mini-pigs and co-transfected with three genes, namely the complement regulatory factors, human decay accelerating factor (hDAF) and human (h) CD59, as well as H-transferase.

Therefore, the objective of the current study was to understand the contribution of transgenic donor cell features to variations in SCNT efficiency. For this purpose, various parameters associated with viability and senescence were compared among four fetal fibroblast cell lines, including 
Table I. Primers used for gene expression analysis.

\begin{tabular}{|c|c|c|c|}
\hline Gene & Primer sequences $\left(5^{\prime}-3^{\prime}\right)$ & Product size (bp) & GenBank accession number \\
\hline Bax & $\begin{array}{l}\text { F: TGCCTCAGGATGCATCTACC } \\
\text { R: AAGTAGAAAAGCGCGACCAC }\end{array}$ & 199 & XM_003127290 \\
\hline$B c l-2$ & $\begin{array}{l}\mathrm{F}: \text { AGGGCATTCAGTGACCTGAC } \\
\text { R: CGATCCGACTCACCAATACC }\end{array}$ & 193 & NM_214285 \\
\hline p53 & $\begin{array}{l}\text { F: CTTTGAGGTGCGTGTTTGTG } \\
\text { R: CGGATCTGGAGGGTGAAATA }\end{array}$ & 192 & NM_213824 \\
\hline GAPDH & $\begin{array}{l}\text { F: GTCGGTTGTGGATCTGACCT } \\
\text { R: TTGACGAAGTGGTCGTTGAG }\end{array}$ & 207 & NM_001206359 \\
\hline
\end{tabular}

morphology, growth rate, mRNA expression and reactive oxygen species (ROS) production.

\section{Materials and methods}

Cell lines and culture procedure. Two fetal fibroblast cell lines were used as controls in the study, originating from LYD or Yucatan mini pigs. LYD cells were transfected with a tetracycline-responsive gene (tetracycline on/off cells). Yucatan cells were transfected with the complement regulatory factors, hDAF and hCD59, as well as H-transferase (triple-transfected cells). Thus, each transfected line was compared with its originating control (LYD and Yucatan). Cells were cultured in Dulbecco's modified Eagle's medium (DMEM; Gibco-BRL, Grand Island, NY, USA) containing $25 \mu \mathrm{M}$ glucose (high glucose medium) plus $10 \%$ fetal bovine serum (FBS; Gibco-BRL). A $130 \mu \mathrm{g} / \mathrm{ml}$ hygromycin B solution from Streptomyces hygroscopious (Sigma-Aldrich, St. Louis, MO, USA) was used for culturing the triple transgenic cell line. Cell cultures were maintained in a 6-well dish and subcultured at a 1:4 ratio once $\sim 90 \%$ confluence was observed. The culture medium was replaced every two days. Assays were always performed in cells from the 9 th population doubling (PD) passage.

Determination of PD times and cell counts. Following the initial seeding, counting was performed once cells reached 95\% confluence. For this purpose, cells were trypsinized, harvested and centrifuged (Hanshin Medical Co., Seoul, Republic of Korea) at 2,000 rpm for $3 \mathrm{~min}$. The pellet was then suspended in DMEM plus 10\% FBS and an aliquot was used for counting with a hemocytometer (Superior, Marienfeld, Germany). Cells were seeded again and the entire aforementioned procedure was repeated twice. Thus, PD times were calculated by averaging the time required for the cell number to double during the exponential growth phase, that is, during the three cell subculture from passage 7 to passage 9 .

Morphological analysis. Phase contrast microscopy (Leica Microsystems, Wetzlar, Germany) was used to assess cell morphology. Examinations were performed on specimens fixed for $5 \mathrm{~min}$ in $4 \%$ paraformaldehyde at room temperature.

Quantitative polymerase chain reaction ( $q P C R)$. Total RNA was extracted from the various cell lines using TRIzol reagent (Invitrogen Life Technologies, Carlsbad, CA, USA), according to the manufacturer's instructions. Complementary DNA (cDNA) was prepared by the reverse transcription of $1 \mu \mathrm{g}$ total RNA using Moloney murine leukemia virus reverse transcriptase (Invitrogen Life Technologies) and random primers (9-mers; Takara Bio, Inc., Shiga, Japan). qPCR (Mx3000P qPCR System; Agilent Technologies, Inc., Santa Clara, USA) was then performed with $1 \mu \mathrm{l}$ cDNA template added to $10 \mu 12 \mathrm{X}$ SYBR Premix Ex Taq (Takara Bio, Inc.) containing specific primers. Amplification was performed for 40 cycles with cycling parameters as follows: Denaturation at $95^{\circ} \mathrm{C}$ for $30 \mathrm{sec}$, annealing at $55^{\circ} \mathrm{C}$ for $30 \mathrm{sec}$ and extension at $72^{\circ} \mathrm{C}$ for $30 \mathrm{sec}$. All primer sequences are presented in Table I. The expression of each target gene was quantified relative to that of the internal control gene, GAPDH. The relative quantification was based upon the comparison of the threshold cycle $(\mathrm{Ct})$ at a constant fluorescence intensity. Relative mRNA expression $(\mathrm{R})$ was calculated using the following equation: $\mathrm{R}=2^{-[\Delta \text { Ctsample }-\Delta \text { Ctcontrol }]}$. To determine the normalized arbitrary value for each gene, values were normalized against GAPDH.

Measurement of ROS. To determine the intracellular content of ROS, cells were incubated with $5 \mu \mathrm{g} / \mathrm{ml} 2^{\prime}, 7^{\prime}$-dichlorodihydrofluorescein diacetate $\left(\mathrm{H}_{2} \mathrm{DCF}-\mathrm{DA}\right.$; Invitrogen Life Technologies) for $30 \mathrm{~min}$ at $37^{\circ} \mathrm{C}$. The acetates of $\mathrm{H}_{2} \mathrm{DCF}-\mathrm{DA}$ were cleaved by intracellular esterases, releasing the reduced fluorescein, which was then converted to dichlorofluorescein in the presence of ROS and detected by fluorescence activated cell sorting (FACS; Becton Dickinson Immunocytometry Systems, San Jose, CA, USA). Dye-loaded cells were trypsinized for $2 \mathrm{~min}$ and the reaction was stopped with phosphate-buffered saline. Living cells were pelleted by centrifugation at $357 \mathrm{x} \mathrm{g}$ for 3 min. Following washing with PBS, cells were resuspended in PBS and FACS analysis was performed using emission and excitation wavelengths of 530 and $480 \mathrm{~nm}$, respectively. The detected levels of DCF fluorescence correlated with the ROS levels. Relative levels were compared between the transfected cell lines and their control counterparts.

Statistical analysis. Quantitative measurements were performed at least three times, with data expressed as the mean \pm standard error of the mean. Comparisons of mean values between various groups were performed by one-way analysis of variance. $\mathrm{P}<0.05$ was considered to indicate a statistically significant difference. 
A

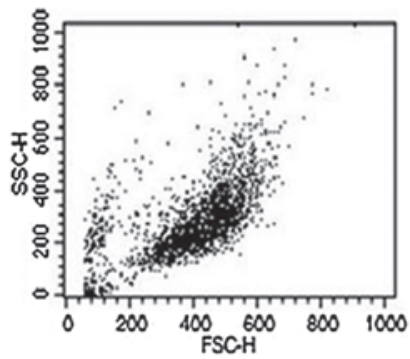

C

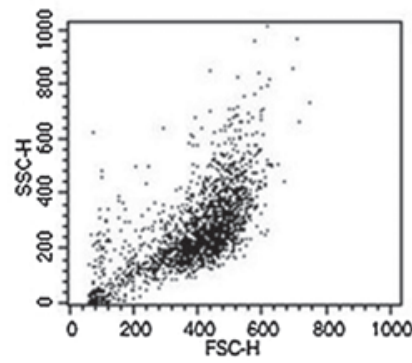

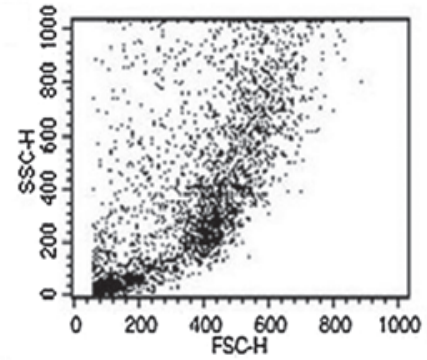

D

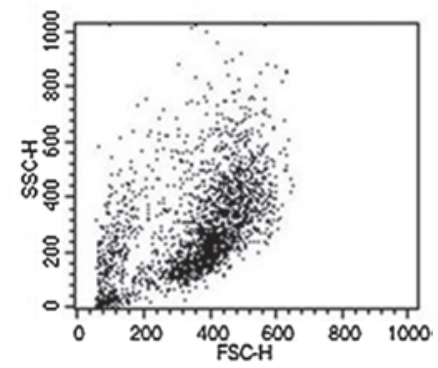

Figure 1. Scatter plots showing cell viability analysis by flow cytometry of fetal fibroblasts. Fibroblasts from (A) LYD crossbred pigs and (B) Yucatan mini-pigs. (C) LYD fibroblasts transfected with a tetracycline on/off gene. (D) Yucatan fibroblasts transfected with three genes. Images were captured during passage 9. X axis, cell density; Y axis, nuclear density. LYD, Landrace x Yorkshire x Duroc.

\section{Results}

Cell proliferation rate. For cells in culture, the mean PD time was correlated with the proliferation rate. Mean PD times were 26, 40, 26 and $66 \mathrm{~h}$ for the LYD, Yucatan, tetracycline on/off and triple transfected cell lines, respectively. Thus, PD times were longer for LYD than for Yucatan originated fibroblasts. Transgenic tetracycline on/off cell lines had a similar mean PD time to non-transfected LYD fibroblasts, whereas triple transfected cell lines required longer $(\mathrm{P}<0.05) \mathrm{PD}$ times than non-transfected Yucatan controls.

Morphological analysis. Based on the phase contrast microscopy observations, fibroblasts from Yucatan mini-pigs were larger than those from LYD crossbred pigs, which was consistent with the FACS analysis results (Fig. 1). Conversely, there were no morphological differences between the transfected and corresponding non-transfected controls, with the exception of cell density, as shown by the PD time calculation 3 days following thawing (Figs. 1 and 2).

Gene-expression analysis. To assess cellular aging and apoptosis at the gene level, the mRNA expression levels of TP53, Bax and $\mathrm{Bcl}-2$ were examined in the cell lines. Initially, Yucatan fibroblasts had significantly lower levels of TP53 mRNA and higher levels of $\mathrm{Bcl}-2 \mathrm{mRNA}$ compared with LYD fibroblasts (Fig. 3A). When comparing tetracycline on/off transgenic cell lines with the original LYD fibroblasts, the former expressed lower levels of $\mathrm{Bcl}-2 \mathrm{mRNA}$, with no differences in TP53 or Bax mRNA expression levels (Fig. 3B). In addition, triple transgenic cell lines exhibited a threefold higher TP53 mRNA expression level and a twofold higher Bax mRNA expression level than the non-transfected Yucatan fibroblasts (Fig. 3C).

ROS production analysis. It has been reported that overproduction of ROS initiates apoptosis (11). Therefore, intracellular ROS levels were evaluated using a DCFH-DA probe (Fig. 4) and FACS analysis (Fig. 5). ROS levels were significantly different depending on cell type and transfection status. The histograms shown correspond to the representative result from three independent experiments (Fig. 5). The rightward shift observed for Yucatan fibroblasts is consistent with more extensive dye oxidation, yielding a relative increase in fluorescence (Fig. 5A). In general, results were consistent with the qualitative differences among cell lines and hence indicated that the two transgenic cell lines exhibited higher intracellular ROS levels when compared with their originating non-transfected cell lines (Fig. 5B and C).

\section{Discussion}

Survival rates of SCNT embryos are reportedly lower in swine than in other domestic species (12). While a number of factors are likely to contribute to the lower success rates, the main reasons for consistent inefficiency of pig cloning remain unclear. A previous study indicated that incomplete remodeling of the donor somatic cell nucleus was a key factor contributing to the low success of SCNT in swines (13). This was in agreement with studies showing that the age, size, gender and type of donor cell may affect the efficiency of SCNT (4,14-16). Moreover, culturing donor cells for long time periods resulted in decreased rates of in vitro embryos and to term development in cattle (17). Altogether, these studies indicate that nuclear donor cell preparation is a crucial step affecting the developmental potential of reconstructed embryos and the successful production of transgenic animals by SCNT. Thus, the present study used four potential donor cell types, based on the breed of origin and transgenic status, and analyzed parameters associated with senescence and apoptosis.

The $B c l-2$ family of proteins comprises members with dual functions with regard to positively or negatively regulating apoptosis. For example, $\mathrm{Bcl}-2$ inhibits apoptosis and proliferation, while $B a x$ is pro-apoptotic and proliferative (18). In addition, p53, a protein involved in the regulation 
A

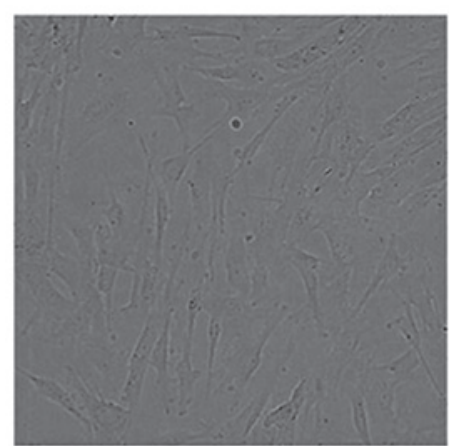

C

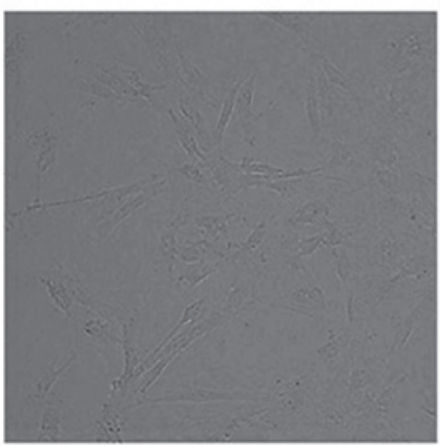

B

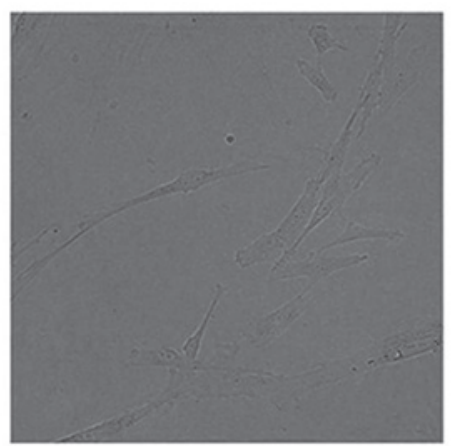

D

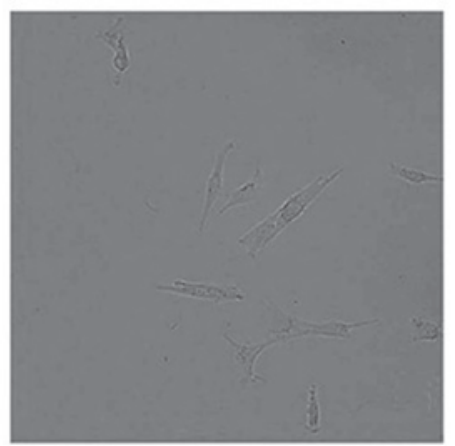

Figure 2. Phase contrast photomicrography of fetal fibroblasts. Fibroblasts from (A) LYD crossbred pigs and (B) Yucatan mini-pigs. (C) LYD fibroblasts transfected with a tetracycline on/off gene. (D) Yucatan fibroblasts transfected with three genes. Images were captured during passage 9. LYD, Landrace x Yorkshire x Duroc. 200X magnification.

of cellular aging, is postulated to inhibit the mammalian target of rapamycin and suppress p21-induced senescence, thus causing quiescence (19). Thus, in the present study, relative expression levels were measured for the aforementioned genes in order to assess cell senescence and/or apoptosis, which may negatively affect the potential of donor cells for SCNT purposes. Notably, the Yucatan cell line exhibited lower expression levels of TP53 mRNA and higher expression levels of $\mathrm{Bcl}-2$ when compared with other cell lines, which correlates with the PD times observed. When compared with LYD fibroblasts, Yucatan cells exhibited a 1.5-fold longer PD time. As for transgenic cells, tetracycline on/off transfected cells exhibited a lower relative expression of antiapoptotic $B c l-2$ when compared with the parental LYD cells, indicating that the transfected cells had a diminished ability to avoid an apoptotic event. By comparison, triple-transfected cell lines exhibited significantly higher expression levels of TP53 and Bax when compared with the parental Yucatan fibroblasts. It was therefore hypothesized that this may enhance the probability of these cells undergoing p53-dependent apoptosis, resulting in Bax-driven induction of mitochondrial permeabilization (20). These observations indicate that donor cell transfection may weaken the defense mechanisms against apoptosis or even enforce pathways leading to programmed cell death while in culture. For example, in a study by $\mathrm{Li}$ and Gao, CD59 transfected cells underwent caspase-3 activation and apoptosis induction (21). While in this study there was no effect on Bax expression levels, an additional study showed that overexpression of CD59 inhibited antiapoptotic $\mathrm{Bcl}-2$ expression (22). These discrepancies are hypothesized to be due to the differences in cell types; HeLa cells (cervical cancer cells) and breast cancer cells were used in these two studies.
At the cellular level, it has been reported that ROS accelerates telomere shortening and induces senescence (23). Notably, ROS levels increase as cells progress towards senescence through various pathways $(24,25)$. In the present study, a rightward shift was observed in the FACS histogram for Yucatan fibroblasts when compared with LYD cells, which is consistent with ROS accumulation potentially due to aging. Thus, decreased antioxidant capacity may account for a decrease in ROS scavenging, contributing to aging-associated cell oxidative damage (26).

The $\mathrm{p} 53$ transcription factor is key in the signal networks of senescence and ROS-induced apoptosis $(27,28)$. In the present study, increased ROS production was observed in transfected cells, indicating that this procedure enhanced aging and apoptosis. In addition, it was reported that p53-mediated senescence (29) and apoptosis (30) are enhanced in modified cells. Thus, the significantly high production of ROS paired with high expression levels of p53 and Bax in triple-transfected cells markedly indicate that ROS-mediated activation of apoptosis may potentially occur via a $p 53$-driven pathway in this cell line. Therefore, ROS may be acting as upstream factors activating TP53, as well as downstream factors targeting p53-mediated Bax activation. The interpretation of the correlation between apoptosis and aging remains controversial. While several studies are consistent with apoptosis being upregulated during senescence in diverse cells, including cardiomyocytes (31), hepatocytes (32) and fibroblasts (33), other studies indicate that oxidative stress does not induce apoptosis in senescent cells (34) and that senescence may attenuate apoptosis (35). The different interpretations may have resulted from the variations in species, strains or cell types used in the respective studies. 
A

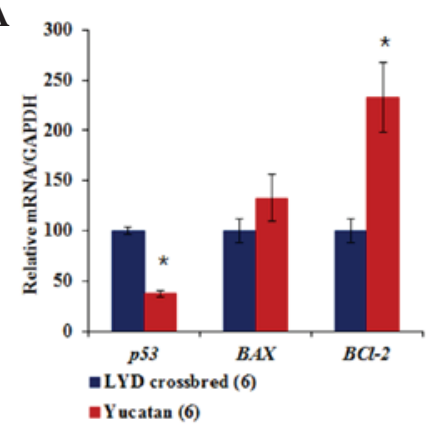

B

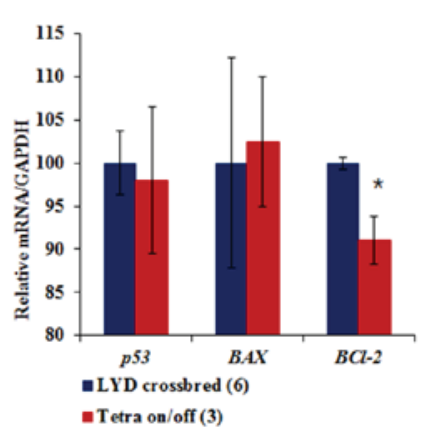

C

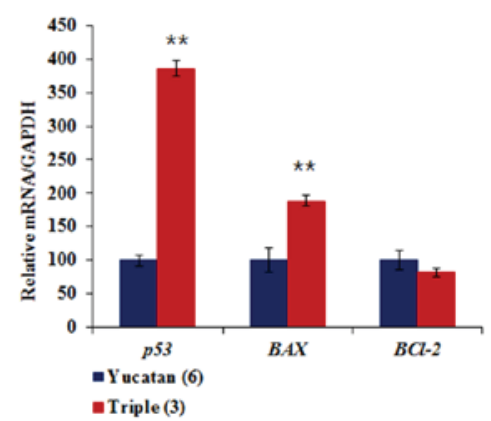

Figure 3. Relative mRNA expression levels of $p 53$, Bax and $B c l-2$ by qPCR analysis. Each bar represents the mean \pm standard deviation. (n), no. of replicates. ${ }^{*} \mathrm{P}<0.01$ and ${ }^{* *} \mathrm{P}<0.05$, where P-values of $(\mathrm{A}),(\mathrm{B})$, and $(\mathrm{C})$ are compared with LYD crossbred, LYD crossbred and Yucatan, respectively. $\mathrm{PPCR}$, quantitative PCR.

A

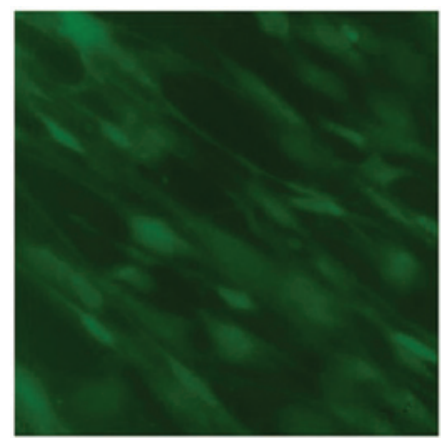

C

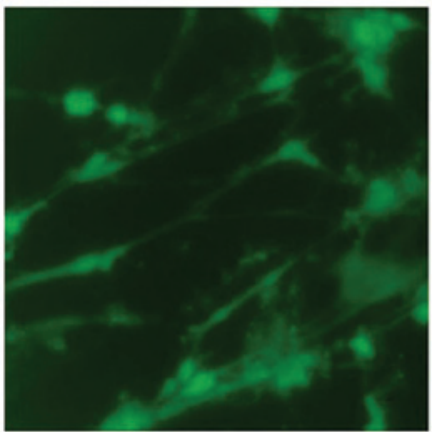

B

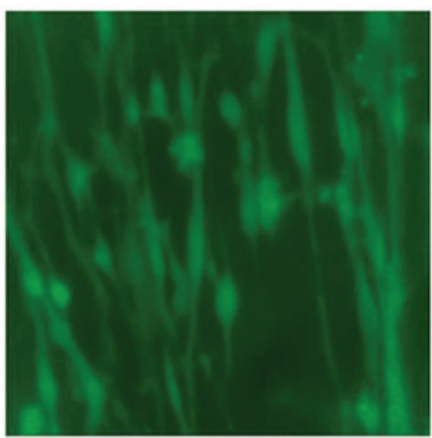

D

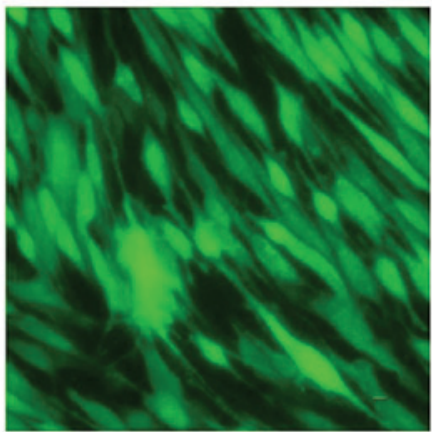

Figure 4. Fluorescent photomicrograph of cultured swine fetal fibroblasts in the 9th passage. (A) LYD, (B) Yucatan and (C) LYD fibroblasts transfected with a tetracycline on/off gene and (D) triple-transfected Yucatan pig fibroblasts. Cells were stained with $\mathrm{H}_{2}$ DCFH-DA during passage 9 to assess ROS formation. LYD, Landrace x Yorkshire x Duroc; $\mathrm{H}_{2} \mathrm{DCFH}-\mathrm{DA}, 2^{\prime}$, '7'-dichlorofluorescin diacetate; ROS, reactive oxygen species.
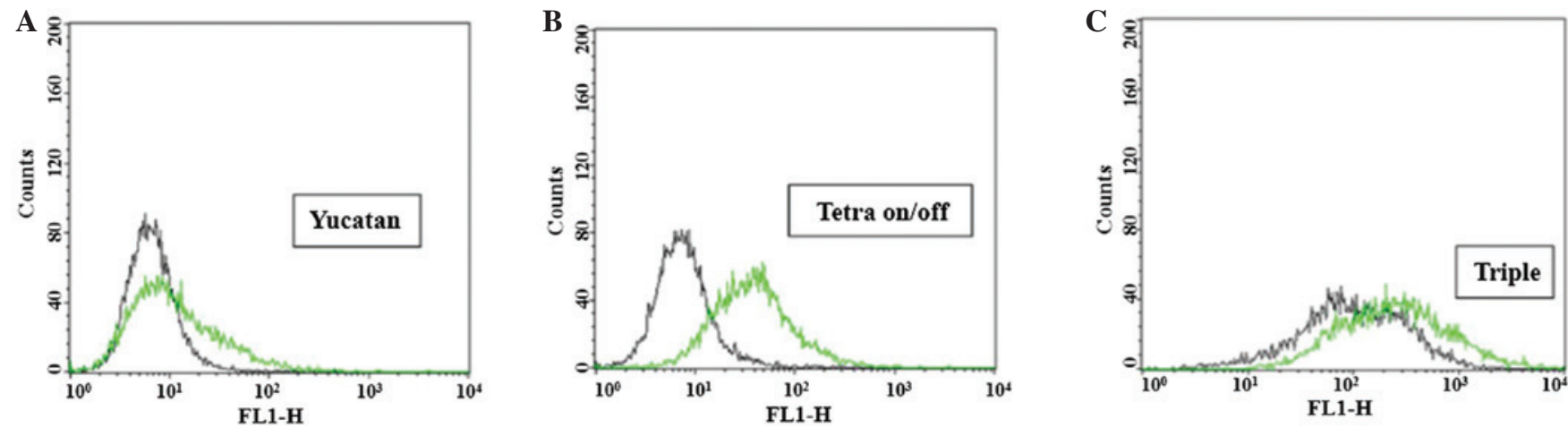

Figure 5. Histograms showing ROS levels (green fluorescence) analyzed using flow cytometry. Results shown are representative of one of three experiments. ROS, reactive oxygen species.

Questions remain with regard to assessing the viability of transfected cell lines for use in SCNT. The effect of transfected donor cells upon reconstructed SCNT embryos have been previously reported (36). In the present study, the average number of blastocyst cells was significantly lower for the embryos reconstructed using transfected fibroblasts. 
In conclusion, the characteristics of individual transgenic cell types and their associations with senescence and apoptosis following transfection may be important determinants of the developmental ability of SCNT embryos. However, further studies are required to clarify the mechanisms involved.

\section{Acknowledgements}

This study was supported by the Basic Science Research Program through the National Research Foundation of Korea, funded by the Ministry of Education, Science and Technology (grant no. 2012004885). The authors would like to thank the Sooam Biotech Research Foundation for the donation of the cell lines (Yucatan fibroblasts and triple-transfected cells).

\section{Reference}

1. Kato Y, Tani T, Sotomaru Y, Kurokawa K, Kato J, Doguchi $\mathrm{H}$, et al: Eight calves cloned from somatic cells of a single adult. Science 282: 2095-2098, 1998.

2. Lee BC, Kim MK, Jang G, Oh HJ, Yuda F, Kim HJ, et al: Dogs cloned from adult somatic cells. Nature 436: 641, 2005.

3. Galli C, Lagutina I, Crotti G, Colleoni S, Turini P, Ponderato N, et al: Pregnancy: a cloned horse born to its dam twin. Nature 424: 635, 2003.

4. Wakayama T and Yanagimachi R: Mouse cloning with nucleus donor cells of different age and type. Mol Reprod Dev 58: 376-383, 2001.

5. Oback B and Wells D: Practical aspects of donor cell selection for nuclear cloning. Cloning Stem Cells 4: 169-174, 2002.

6. Batchelder CA, Hoffert KA, Bertolini M, Moyer AL, Mason JB, Petkov SG, et al: Effect of the nuclear-donor cell lineage, type, and cell donor on development of somatic cell nuclear transfer embryos in cattle. Cloning Stem Cells 7: 238-254, 2005.

7. Kubota C, Yamakuchi H, Todoroki J, Mizoshita K, Tabara N, Barber M and Yang X: Six cloned calves produced from adult fibroblast cells after long-term culture. Proc Natl Acad Sci USA 97: 990-995, 2000.

8. Roh S, Shim H, Hwang WS and Yoon JT: In vitro development of green fluorescent protein (GFP) transgenic bovine embryos after nuclear transfer using different cell cycles and passages of fetal fibroblasts. Reprod Fertil Dev 12: 1-6, 2000.

9. Zhu Z, Zheng T, Lee CG, Homer RJ and Elias JA: Tetracycline-controlled transcriptional regulation systems: advances and application in transgenic animal modeling. Semin Cell Dev Biol 13: 121-128, 2002.

10. Ramírez P, Montoya MJ, Ríos A, García Palenciano C, Majado M, Chávez R, et al: Prevention of hyperacute rejection in a model of orthotopic liver xenotransplantation from pig to baboon using polytransgenic pig livers (CD55, CD59, and H-transferase). Transplant Proc 37: 4103-4106, 2005.

11. Circu ML and Aw TY: Reactive oxygen species, cellular redox systems, and apoptosis. Free Radic Biol Med 48: 749-762, 2010.

12. Pratt SL, Sherrer ES, Reeves DE and Stice SL: Factors influencing the commercialisation of cloning in the pork industry. Soc Reprod Fertil Suppl 62: 303-315, 2006.

13. Zhao J, Whyte J and Prather RS: Effect of epigenetic regulation during swine embryogenesis and on cloning by nuclear transfer. Cell Tissue Res 341: 13-21, 2010.

14. Yang F, Hao R, Kessler B, Brem G, Wolf E and Zakhartchenko V: Rabbit somatic cell cloning: effects of donor cell type, histone acetylation status and chimeric embryo complementation. Reproduction 133: 219-230, 2007.

15. Hosseini SM, Moulavi F, Foruzanfar M, Hajian M, Abedi P, Rezazade-Valojerdi M, et al: Effect of donor cell type and gender on the efficiency of in vitro sheep somatic cell cloning. Small Rumin Res 78: 162-168, 2008.
16. Zhang YH, Song ES, Kim ES, Cong PQ, Lee SH, Lee JW, et al: Effects of donor cell passage, size and type on development of porcine embryos derived from somatic cell nuclear transfer. Asian-Aust J Anim Sci 22: 194-200, 2009.

17. Hill JR, Winger QA, Long CR, Looney CR, Thompson JA and Westhusin ME: Development rates of male bovine nuclear transfer embryos derived from adult and fetal cells. Biol Reprod 62: 1135-1140, 2000.

18. Zinkel S, Gross A and Yang E: BCL2 family in DNA damage and cell cycle control. Cell Death Differ 13: 1351-1359, 2006.

19. Leontieva OV and Blagosklonny MV: DNA damaging agents and p53 do not cause senescence in quiescent cells, while consecutive re-activation of mTOR is associated with conversion to senescence. Aging (Albany NY) 2: 924-935, 2010.

20. Basu A and Haldar S: The relationship between BcI2, Bax and p53: consequences for cell cycle progression and cell death. Mol Hum Reprod 4: 1099-1109, 1998.

21. Li XP and Gao MH: Effect of peptide seals specific to CD59 on the expression of apoptosis-related genes in HeLa cells. Xi Bao Yu Fen Zi Mian Yi Xue Za Zhi 24: 20-22, 2008 (In Chinese).

22. Li B, Chu X, Gao M and Xu Y: The effects of CD59 gene as a target gene on breast cancer cells. Cell Immunol 272: 61-70, 2011.

23. von Zglinicki T: Oxidative stress shortens telomeres. Trends Biochem Sci 27: 339-344, 2002.

24. Passos JF and Von Zglinicki T: Oxygen free radicals in cell senescence: are they signal transducers? Free Radic Res 40: 1277-1283, 2006.

25. Terada LS: Specificity in reactive oxidant signaling: think globally, act locally. J Cell Biol 174: 615-623, 2006.

26. Lu CY, Lee HC, Fahn HJ and Wei YH: Oxidative damage elicited by imbalance of free radical scavenging enzymes is associated with large-scale mtDNA deletions in aging human skin. Mutat Res 423: 11-21, 1999.

27. Vigneron A and Vousden KH: p53, ROS and senescence in the control of aging. Aging (Albany NY) 2: 471-474, 2010.

28. Li PF, Dietz R and von Harsdorf R: p53 regulates mitochondrial membrane potential through reactive oxygen species and induces cytochrome c-independent apoptosis blocked by Bcl-2. EMBO J 18: 6027-6036, 1999.

29. Maier B, Gluba W, Bernier B, Turner T, Mohammad K, Guise T, et al: Modulation of mammalian life span by the short isoform of p53. Genes Dev 18: 306-319, 2004.

30. Tyner SD, Venkatachalam S, Choi J, Jones S, Ghebranious N, Igelmann $\mathrm{H}$, et al: p53 mutant mice that display early ageing-associated phenotypes. Nature 415: 45-53, 2002.

31. Kajstura J, Cheng W, Sarangarajan R, Li P, Li B, Nitahara JA, et al: Necrotic and apoptotic myocyte cell death in the aging heart of Fischer 344 rats. Am J Physiol 271: H1215-H1228, 1996.

32. Muskhelishvili L, Hart RW, Turturro A and James SJ: Age-related changes in the intrinsic rate of apoptosis in livers of diet-restricted and ad libitum-fed B6C3F1 mice. Am J Pathol 147: 20-24, 1995.

33. Kujoth GC, Hiona A, Pugh TD, Someya S, Panzer K, Wohlgemuth SE, et al: Mitochondrial DNA mutations, oxidative stress, and apoptosis in mammalian aging. Science 309: 481-484, 2005.

34. Gansauge S, Gansauge F, Gause H, Poch B, Schoenberg MH and Beger HG: The induction of apoptosis in proliferating human fibroblasts by oxygen radicals is associated with a p53- and p21WAF1CIP1 induction. FEBS Lett 404: 6-10, 1997.

35. Xiao ZQ, Moragoda L, Jaszewski R, Hatfield JA, Fligiel SE and Majumdar AP: Aging is associated with increased proliferation and decreased apoptosis in the colonic mucosa. Mech Ageing Dev 122: 1849-1864, 2001.

36. Kurome M, Ishikawa T, Tomii R, Ueno S, Shimada A, Yazawa H and Nagashima H: Production of transgenic and non-transgenic clones in miniature pigs by somatic cell nuclear transfer. J Reprod Dev 54: 156-63, 2008. 